\title{
Modeling the Effect of Human Mobility on Dengue Transmission
}

\author{
Bentara Wadu Mesthrige Nadi Madushani De Silva ${ }^{1,}$, , Shyam Sanjeewa Nishantha Perera ${ }^{1}$, \\ Naleen Chaminda Ganegoda ${ }^{2}$ \\ ${ }^{1}$ Department of Mathematics, University of Colombo, Colombo, Sri Lanka \\ ${ }^{2}$ Department of Mathematics, University of Sri Jayewardenepura, Nugegoda, Sri Lanka
}

Email address:

madushani.ns@gmail.com (B. W. M. N. M. De Silva), ssnp@maths.cmb.ac.lk (S. S. N. Perera), naleen@sjp.ac.lk (N. C. Ganegoda)

${ }^{*}$ Corresponding author

\section{To cite this article:}

Bentara Wadu Mesthrige Nadi Madushani De Silva, Shyam Sanjeewa Nishantha Perera, Naleen Chaminda Ganegoda. Modeling the Effect of Human Mobility on Dengue Transmission. Applied and Computational Mathematics. Vol. 5, No. 4, 2016, pp. 169-176.

doi: 10.11648/j.acm.20160504.11

Received: June 14, 2016; Accepted: July 18, 2016; Published: August 3, 2016

\begin{abstract}
Dengue is one of the most prevalent viruses transmitted by mosquitoes where increasing incidence and severity claim severe social burden. This virus is common throughout the tropics and subtropics. Dengue is a virus which propagated during the day and thus the mobility of humans can cause it to spread quickly. In this paper, we introduce mobility of humans between two neighboring areas into a mathematical model for the transmission of dengue. Dengue transmission is modeled using the classical SIR model. Simulations have been carried under four cases to compare the impact of human mobility to propagate the dengue disease. These cases are based on existence of dengue and human mobility direction regarding neighboring area. Numerical simulations are carried out using Matlab routine ode 45.
\end{abstract}

Keywords: Dengue Disease, Mathematical Model, Human Mobility

\section{Introduction}

Dengue fever is reported throughout the nineteenth and early twentieth centuries in the America, Southern Europe, North Africa, the eastern Mediterranean, Asia, Australia, and on various islands in the Indian Ocean, the south, central Pacific and the Caribbean. It is named in 1779 and the first recorded epidemics is occurred in Asia, Africa and North America in the 1780s [12]. It is caused by family of viruses that is transmitted by Aedes Aegypti mosquitoes. This disease is classified into three forms; dengue fever (DF), dengue hemorrhagic fever (DHF) and dengue shock syndrome (DSS). DF is a flu-like illness that affects infants, young children and adults, but seldom causes death. DHF is more severe and associated with loss of appetite, vomiting, high fever, headache and abdominal pain. During dengue epidemics in the Philippines and Thailand, DHF is first identified in the 1950s [13]. The dengue flavivirus occurs in four different serotypes, namely DEN-1, DEN-2, DEN-3 and DEN-4. Contracting one form of dengue fever provides lifelong immunity from that serotype, but not from the other serotypes. When a female Aedes mosquito bites a person infected with dengue, the virus incubates in the insect's body for 8-11 days, after which the mosquito can spread the disease to other humans for the remainder of the insect's life span which is between 15 and 65 days $[1,3]$.

Dengue is currently the most prevalent mosquito-borne viral disease affecting humans with 50-100 million estimated cases of dengue fever and 250,000 to 500,000 cases of the more severe dengue hemorrhagic fever/dengue shock syndrome each year, with about 20,000 deaths [5]. The World Health Organization estimates that 3.9 billion people in 128 countries are at risk for dengue [13]. During the past few years, the characteristics of dengue in Sri Lanka appear to have changed [18]. For instance, a decade ago, children were predominantly affected, but in recent years, increasing numbers of adult dengue patients have been seen, with both significant morbidity and increasing number of adult deaths due to dengue. Therefore, it is very important to investigate, how this virus spreads while considering their age, gender and 
other demographic factors.

Mosquitoes carrying dengue typically bite during the daytime, particularly around sunrise and sunset. During the daytime there is high mobility of human because humans move for work, school, shopping and general needs. Since dengue mosquitoes usually only fly short distances $(50-200 \mathrm{~m})$ mosquito mobility does not make a significant influence to spread of dengue in between regions/cities. However, human mobility plays a vital role in the spread of dengue fever in inter region. This paper is an attempt to investigate and model the impact of human mobility to spread of dengue in two neighboring cities or regions.

\section{The Mathematical Model}

Even though vector (mosquito)-transmitted diseases have been around for centuries, we are still trying to determine mathematical models and identify control measure for many of these diseases. History of using epidemiological model goes 1760. The great mathematician, Daniel Bernoulli, formulated epidemiological model with the aim of evaluating the impact of variolation on human life expectancy [6]. In 1927, Kermack and Mckendrick introduced the threshold theorem, which is one of the key concepts in epidemiology [6].

Modeling the spread of Dengue with ordinary differential equations began in the early 1990s. In [8], the authors look at modeling two specific outbreaks of dengue in South America, one with control of the vectors and one without. Santos et al [9] use a cellular automata approach to model the dynamics of dengue spread. In [10], a differential equation model for dengue with susceptible, exposed, and infected mosquitoes and humans are presented. These models are the basis of most of developed models by adding further; many additional factors such as climate, breeding conditions, locality and etc. In [11], the authors introduce a partial differential equation with aquatic, susceptible, and infectious mosquitoes with spatial diffusion considered only in a small locality, no large scale spread of humans is considered in this model. Cross Wavelet power spectrum and Cohency analysis are performed identify dengue spread among Colombo city and neighbouring cities [14]. In [16], the two patch system are developed to identify the movement of human mobility in between two patches. In [17], a conceptual model to evaluate the importance of variation in exposure due to individual human movement for pathogen transmission, focusing on mosquito-borne dengue virus.

\section{The Model formation}

In this paper, we present a mathematical model for the transmission of dengue which considers the mobility of the human population between two neighboring regions.

The birth and death for the human population are omitted. The human population is divided into three classes namely, susceptible, infected, and recovered. A susceptible human is the person who is not immune and not infected [1]. An infected human is the person who is got dengue virus from the infected vector [3]. While, a recovered person is the infected person after the viremia stage until after they recovered from dengue virus infection [3]. The vector population is divided into two classes as susceptible and infected. Since life time of the vector population is very low compared to human life time, recovered class of vectors is not considered. In addition, no incubation period is incorporated into this model.

Let $S_{C_{1}}^{H}(t)$ be the number of susceptible humans in region $C_{1}$ at time $t, I_{C_{1}}^{H}(t)$ be the number of infected humans in region $C_{1}$ at time $t, R_{C_{1}}^{H}(t)$ be the number of removed humans in $C_{1}$ at time $t, S_{C_{1}}^{V}(t)$ be the number of susceptible mosquitoes in region $C_{1}$ at time $t, I_{C_{1}}^{V}(t)$ be the number of infected mosquitoes in region $C_{1}$ at time $t, S_{C_{2}}^{H}(t)$ be the number of susceptible humans in region $C_{2}$ at time $t$, $I_{C_{2}}^{H}(t)$ be the number of infected humans in region $C_{2}$ at time $t, R_{C_{2}}^{H}(t)$ be the number of removed humans in $C_{2}$ at time $t, S_{C_{2}}^{V}(t)$ be the number of susceptible mosquitoes in region $C_{2}$ at time $t, I_{C_{2}}^{V}(t)$ be the number of infected mosquitoes region $C_{2}$ at time $t$. The transmission models of dengue with human mobility between two neighboring regions are described by system of differential equations. System (1-5) describes the dynamics of dengue in region $C_{1}$.

In model, $\alpha_{12} S_{C_{1}}^{H}$ and $\alpha_{21} S_{C_{2}}^{H}$ denote the mobility of susceptible human from region $C_{1}$ to region $C_{2}$ and the mobility of susceptible human from region $C_{2}$ to region $C_{1}$ respectively. Let $\alpha_{12}, \beta_{12}, \gamma_{12}$ denote the rate of the mobility from region $C_{1}$ to $C_{2}$ of susceptible, infected and recovered individuals respectively. In addition, let $\alpha_{21}, \beta_{21}, \gamma_{21}$ denote the rate of the mobility from region $C_{2}$ to $C_{1}$ of susceptible, infected and recovered individuals respectively. Susceptible human in region $C_{1}\left(S_{C_{1}}^{H}-\alpha_{12} S_{C_{1}}^{H}+\alpha_{21} S_{C_{2}}^{H}\right)$ have the probability of being infected with dengue virus at a rate $\frac{b_{C_{1}} \beta_{C_{1}} I_{C_{1}}^{V}}{N_{1}}$, where $N_{1}=N_{C_{1}}^{H}-\alpha_{12} S_{C_{1}}^{H}-\beta_{12} I_{C_{1}}^{H}-\gamma_{12} R_{C_{1}}^{H}+\alpha_{21} S_{C_{2}}^{H}+\beta_{21} I_{C_{2}}^{H}+\gamma_{21} R_{C_{2}}^{H}$. Let $b_{C_{1}}$ and $\beta_{C_{1}}$ denote the bitting rate of the vector population in region $C_{1}$ and the transmission probability from vector to human in region $C_{1}$. Members of the human population in the region $C_{1}$ that recover their health after infected are $\gamma_{C_{1}}\left(I_{C_{1}}^{H}-\beta_{12} I_{C_{1}}^{H}+\beta_{21} I_{C_{2}}^{H}\right)$. The mobility of infected human from region $C_{1}$ to region $C_{2}$ and the mobility of infected human from region $C_{2}$ to region $C_{1}$ are represented by $\beta_{12} I_{C_{1}}^{H}$ and $\beta_{21} I_{C_{2}}^{H}$. The mobility of recovered human from region $C_{1}$ to region $C_{2}$ and the mobility of recovered human from region $C_{2}$ to region $C_{1}$ are represented by $\gamma_{12} R_{C_{1}}^{H}$ and $\gamma_{21} R_{C_{2}}^{H}$. Birth of the mosquito to the susceptible compartment 
is $\lambda_{V C_{1}} N_{C_{1}}^{V}$. The number of deaths among the susceptible mosquito population is $\lambda_{V C_{1}} S_{C_{1}}^{V}$ at any given time and the number of deaths among the infected mosquito population is $\lambda_{V C_{1}} I_{C_{1}}^{V}$. Susceptible mosquito in region have the probability of being infected with dengue virus at a rate $\frac{b_{C_{1}} \beta_{V C_{1}}\left(I_{C_{1}}^{H}-\beta_{12} I_{C_{1}}^{H}+\beta_{21} I_{C_{2}}^{H}\right)}{N_{1}}$. Let $\beta_{V C_{1}}$ denotes the transmission probability from human to vector in region $C_{1}$. System (6-10) also describes the dynamics of dengue in region $C_{2}$. Similary, this system also can be described as above. In system (6-10), let $b_{C_{2}}, \beta_{C_{2}}, \gamma_{C_{2}}, \beta_{V C_{2}}$ and $\lambda_{V C_{2}}$ denote bitting rate of the vector population in region $C_{2}$, transmission probability from vector to human in region $C_{2}$, recovery rate of humans in region $C_{2}$, transmission probability from human to vector in region $C_{2}$ and birth/death rate in the vector population in region $C_{2}$ respectively. Also $N_{2}$ denotes $N_{C_{2}}^{H}+\alpha_{12} S_{C_{1}}^{H}+\beta_{12} I_{C_{1}}^{H}+\gamma_{12} R_{C_{1}}^{H}-\alpha_{21} S_{C_{2}}^{H}-\beta_{21} I_{C_{2}}^{H}-\gamma_{21} R_{C_{2}}^{H}$.

The systematic diagram for dengue transmission process is shown in Figure 1.

Region $C_{1}$

Region $C_{2}$

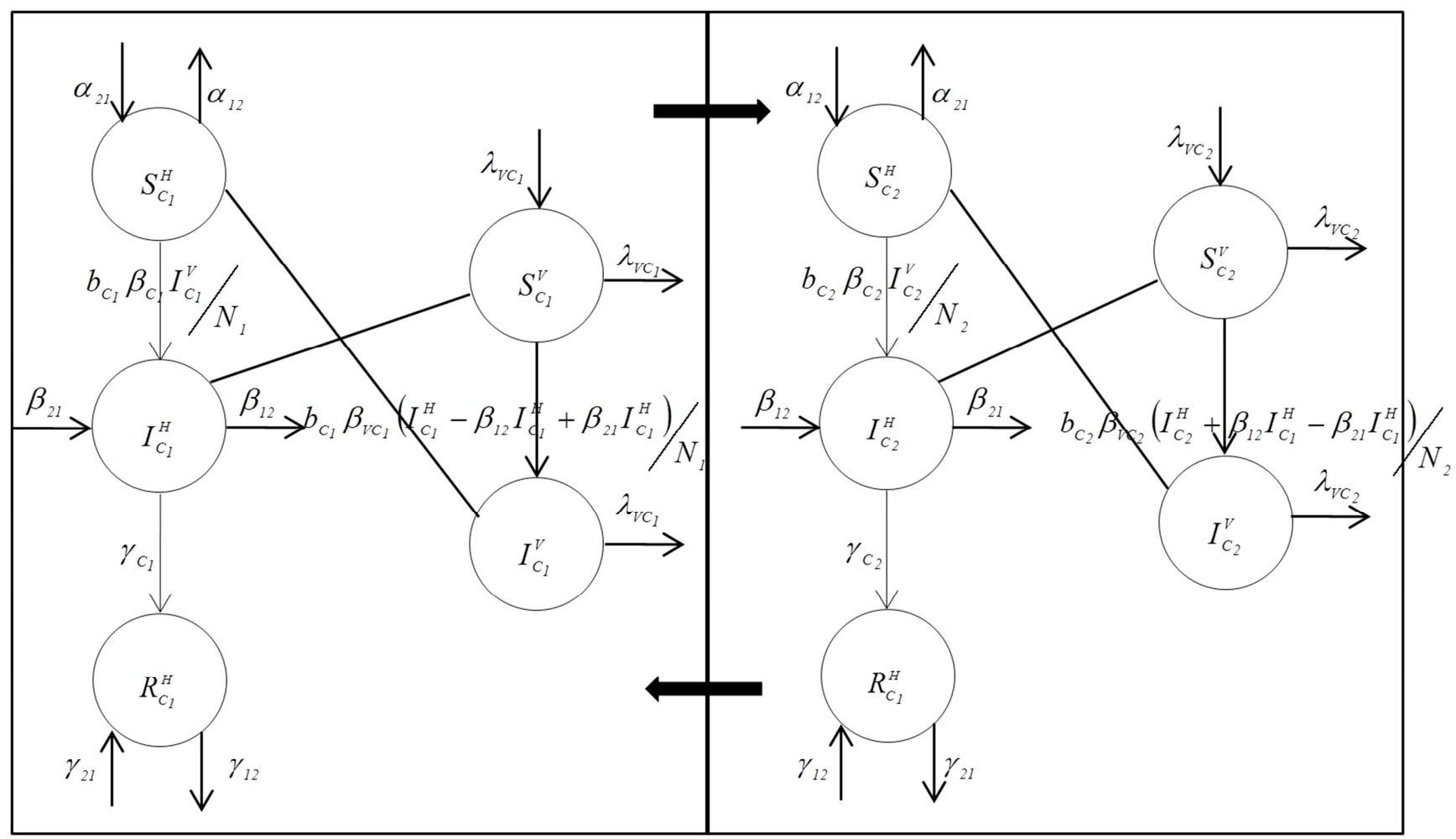

Figure 1. A Systematic diagram of model.

$$
\begin{gathered}
\frac{d S_{C_{1}}^{H}}{d t}=-\left(\frac{b_{C_{1}} \beta_{C_{1}} I_{C_{1}}^{V}\left(S_{C_{1}}^{H}-\alpha_{12} S_{C_{1}}^{H}+\alpha_{21} S_{C_{2}}^{H}\right)}{N_{C_{1}}^{H}-\alpha_{12} S_{C_{1}}^{H}-\beta_{12} I_{C_{1}}^{H}-\gamma_{12} R_{C_{1}}^{H}+\alpha_{21} S_{C_{2}}^{H}+\beta_{21} I_{C_{2}}^{H}+\gamma_{21} R_{C_{2}}^{H}}\right)-\alpha_{12} S_{C_{1}}^{H}+\alpha_{21} S_{C_{2}}^{H} \\
\frac{d I_{C_{1}}^{H}}{d t}=\left(\frac{b_{C_{1}} \beta_{C_{1}} I_{C_{1}}^{V}\left(S_{C_{1}}^{H}-\alpha_{12} S_{C_{1}}^{H}+\alpha_{21} S_{C_{2}}^{H}\right)}{N_{C_{1}}^{H}-\alpha_{12} S_{C_{1}}^{H}-\beta_{12} I_{C_{1}}^{H}-\gamma_{12} R_{C_{1}}^{H}+\alpha_{21} S_{C_{2}}^{H}+\beta_{21} I_{C_{2}}^{H}+\gamma_{21} R_{C_{2}}^{H}}\right)-\gamma_{C_{1}}\left(I_{C_{1}}^{H}-\beta_{12} I_{C_{1}}^{H}+\beta_{21} I_{C_{2}}^{H}\right)-\beta_{12} I_{C_{1}}^{H}+\beta_{21} I_{C_{2}}^{H} \\
\frac{d R_{C_{1}}^{H}}{d t}=\gamma_{C_{1}}\left(I_{C_{1}}^{H}-\beta_{12} I_{C_{1}}^{H}+\beta_{21} I_{C_{2}}^{H}\right)-\gamma_{12} R_{C_{1}}^{H}+\gamma_{21} R_{C_{2}}^{H} \\
\frac{d S_{C_{1}}^{V}}{d t}=\lambda_{V C_{1}} N_{C_{1}}^{V}-\left(\frac{b_{C_{1}} \beta_{V C_{1}}\left(I_{C_{1}}^{H}-\beta_{12} I_{C_{1}}^{H}+\beta_{21} I_{C_{2}}^{H}\right) S_{C_{1}}^{V}}{N_{C_{1}}^{H}-\alpha_{12} S_{C_{1}}^{H}-\beta_{12} I_{C_{1}}^{H}-\gamma_{12} R_{C_{1}}^{H}+\alpha_{21} S_{C_{2}}^{H}+\beta_{21} I_{C_{2}}^{H}+\gamma_{21} R_{C_{2}}^{H}}\right)-\lambda_{V C_{1}} S_{C_{1}}^{V}
\end{gathered}
$$




$$
\begin{aligned}
& \frac{d I_{C_{1}}^{V}}{d t}=\left(\frac{b_{C_{1}} \beta_{V C_{1}}\left(I_{C_{1}}^{H}-\beta_{12} I_{C_{1}}^{H}+\beta_{21} I_{C_{2}}^{H}\right) S_{C_{1}}^{V}}{N_{C_{1}}^{H}-\alpha_{12} S_{C_{1}}^{H}-\beta_{12} I_{C_{1}}^{H}-\gamma_{12} R_{C_{1}}^{H}+\alpha_{21} S_{C_{2}}^{H}+\beta_{21} I_{C_{2}}^{H}+\gamma_{21} R_{C_{2}}^{H}}\right)-\lambda_{V C_{1}} I_{C_{1}}^{V} \\
& \frac{d S_{C_{2}}^{H}}{d t}=-\left(\frac{b_{C_{2}} \beta_{C_{2}} I_{C_{2}}^{V}\left(S_{C_{2}}^{H}+\alpha_{12} S_{C_{1}}^{H}-\alpha_{21} S_{C_{2}}^{H}\right)}{N_{C_{2}}^{H}+\alpha_{12} S_{C_{1}}^{H}+\beta_{12} I_{C_{1}}^{H}+\gamma_{12} R_{C_{1}}^{H}-\alpha_{21} S_{C_{2}}^{H}-\beta_{21} I_{C_{2}}^{H}-\gamma_{21} R_{C_{2}}^{H}}\right)+\alpha_{12} S_{C_{1}}^{H}-\alpha_{21} S_{C_{2}}^{H} \\
& \frac{d I_{C_{2}}^{H}}{d t}=\left(\frac{b_{C_{2}} \beta_{C_{2}} I_{C_{2}}^{V}\left(S_{C_{2}}^{H}+\alpha_{12} S_{C_{1}}^{H}-\alpha_{21} S_{C_{2}}^{H}\right)}{N_{C_{2}}^{H}+\alpha_{12} S_{C_{1}}^{H}+\beta_{12} I_{C_{1}}^{H}+\gamma_{12} R_{C_{1}}^{H}-\alpha_{21} S_{C_{2}}^{H}-\beta_{21} I_{C_{2}}^{H}-\gamma_{21} R_{C_{2}}^{H}}\right)-\gamma_{C_{2}}\left(I_{C_{2}}^{H}+\beta_{12} I_{C_{1}}^{H}-\beta_{21} I_{C_{2}}^{H}\right)+\beta_{12} I_{C_{1}}^{H}-\beta_{21} I_{C_{2}}^{H} \\
& \frac{d R_{C_{2}}^{H}}{d t}=\gamma_{C_{2}}\left(I_{C_{2}}^{H}+\beta_{12} I_{C_{1}}^{H}-\beta_{21} I_{C_{2}}^{H}\right)+\gamma_{12} R_{C_{1}}^{H}-\gamma_{21} R_{C_{2}}^{H} \\
& \frac{d S_{C_{2}}^{V}}{d t}=\lambda_{V C_{2}} N_{C_{2}}^{V}-\left(\frac{b_{C_{2}} \beta_{V C_{2}}\left(I_{C_{2}}^{H}+\beta_{12} I_{C_{1}}^{H}-\beta_{21} I_{C_{2}}^{H}\right) S_{C_{2}}^{V}}{N_{C_{2}}^{H}+\alpha_{12} S_{C_{1}}^{H}+\beta_{12} I_{C_{1}}^{H}+\gamma_{12} R_{C_{1}}^{H}-\alpha_{21} S_{C_{2}}^{H}-\beta_{21} I_{C_{2}}^{H}-\gamma_{21} R_{C_{2}}^{H}}\right)-\lambda_{V C_{2}} S_{C_{2}}^{V} \\
& \frac{d I_{C_{2}}^{V}}{d t}=\left(\frac{b_{C_{2}} \beta_{V C_{2}}\left(I_{C_{2}}^{H}+\beta_{12} I_{C_{1}}^{H}-\beta_{21} I_{C_{2}}^{H}\right) S_{C_{2}}^{V}}{N_{C_{2}}^{H}+\alpha_{12} S_{C_{1}}^{H}+\beta_{12} I_{C_{1}}^{H}+\gamma_{12} R_{C_{1}}^{H}-\alpha_{21} S_{C_{2}}^{H}-\beta_{21} I_{C_{2}}^{H}-\gamma_{21} R_{C_{2}}^{H}}\right)-\lambda_{V C_{2}} I_{C_{2}}^{V}
\end{aligned}
$$

With the condition,

$$
S_{C_{1}}^{H}+I_{C_{1}}^{H}+R_{C_{1}}^{H}+S_{C_{2}}^{H}+I_{C_{2}}^{H}+R_{C_{2}}^{H}=N_{C_{1}}^{H}+N_{C_{2}}^{H}=N, S_{C_{1}}^{V}+I_{C_{1}}^{V}=N_{C_{1}}^{V} \text { and } S_{C_{2}}^{V}+I_{C_{2}}^{V}=N_{C_{2}}^{V}
$$

where, $N_{C_{1}}^{H}$ is the total human population in region $C_{1}$,

$N_{C_{2}}^{H}$ is the total human population in region $C_{2}$,

$N$ is the total human population of region $C_{1}$ and $C_{2}$,

$N_{C_{1}}^{V}$ is the total mosquito population in region $C_{1}$,

$N_{C_{2}}^{V}$ is the total mosquito population in region $C_{2}$.

We assume, both human population of the two neighboring region and vector population are constant in both region. We normalize (1-5) and (6-10) by letting,

$$
\begin{aligned}
& S_{C_{1}}^{H^{*}}=\frac{S_{C_{1}}^{H}}{N} I_{C_{1}}^{H^{*}}=\frac{I_{C_{1}}^{H}}{N} \quad R_{C_{1}}^{H^{*}}=\frac{R_{C_{1}}^{H}}{N} \quad S_{C_{1}}^{V^{*}}=\frac{S_{C_{1}}^{V}}{N} \quad I_{C_{1}}^{V^{*}}=\frac{I_{C_{1}}^{V}}{N} \\
& S_{C 2}^{H^{*}}=\frac{S_{C_{2}}^{H}}{N} I_{C_{2}}^{H^{*}}=\frac{I_{C_{2}}^{H}}{N} \quad R_{C 2}^{H^{*}}=\frac{R_{C_{2}}^{H}}{N} \quad S_{C 2}^{V^{*}}=\frac{S_{C_{2}}^{V}}{N} \quad I_{C 2}^{V^{*}}=\frac{I_{C_{2}}^{V}}{N} .
\end{aligned}
$$

Dropping the starts and rearranging the terms system (1-5) and (6-10) read as

$$
\begin{gathered}
\frac{d S_{C_{i}}^{H}}{d t}=-\left(\frac{b_{C_{i}} \beta_{C_{i}} n_{C_{i}} I_{C_{i}}^{V}\left(S_{C_{i}}^{H}+(-1)^{i}\left(\alpha_{12} S_{C_{1}}^{H}-\alpha_{21} S_{C_{2}}^{H}\right)\right)}{N_{C_{i}}^{H}+\sum_{j=1, j \neq i}^{2} \sum_{i=1}^{2}\left((-1)^{i}\left(\alpha_{i j} S_{C_{i}}^{H}+\beta_{i j} I_{C_{i}}^{H}+\gamma_{i j} R_{C_{i}}^{H}\right)\right)}\right)+(-1)^{i}\left(\alpha_{12} S_{C_{1}}^{H}-\alpha_{21} S_{C_{2}}^{H}\right) \\
\frac{d I_{C_{i}}^{H}}{d t}=\left(\frac{b_{C_{i}} \beta_{C_{i}} n_{C_{i}} I_{C_{i}}^{V}\left(S_{C_{i}}^{H}+(-1)^{i}\left(\alpha_{12} S_{C_{1}}^{H}-\alpha_{21} S_{C_{2}}^{H}\right)\right)}{N_{C_{i}}^{H}+\sum_{j=1, j \neq i}^{2} \sum_{i=1}^{2}\left((-1)^{i}\left(\alpha_{i j} S_{C_{i}}^{H}+\beta_{i j} I_{C_{i}}^{H}+\gamma_{i j} R_{C_{i}}^{H}\right)\right)}\right)-\gamma_{C_{i}}\left(I_{C_{i}}^{H}+(-1)^{i}\left(\beta_{12} I_{C_{1}}^{H}-\beta_{21} I_{C_{2}}^{H}\right)\right)+(-1)^{i}\left(\beta_{12} I_{C_{1}}^{H}-\beta_{21} I_{C_{2}}^{H}\right)
\end{gathered}
$$




$$
\begin{aligned}
& \frac{d R_{C_{i}}^{H}}{d t}=\gamma_{C_{i}}\left(I_{C_{i}}^{H}+(-1)^{i}\left(\beta_{12} I_{C_{1}}^{H}-\beta_{21} I_{C_{2}}^{H}\right)\right)+(-1)^{i}\left(\gamma_{12} R_{C_{1}}^{H}-\gamma_{21} R_{C_{2}}^{H}\right) \\
& \frac{d S_{C_{i}}^{V}}{d t}=\lambda_{V C_{i}}-\lambda_{V C_{i}} S_{C_{i}}^{V}-b_{c_{i}} \beta_{V C_{i}}\left(\frac{\left(I_{C_{i}}^{H}+(-1)^{i}\left(\beta_{12} I_{C_{1}}^{H}-\beta_{21} I_{C_{2}}^{H}\right)\right) S_{C_{i}}^{V}}{N_{C_{i}}^{H}+\sum_{j=1, j \neq i}^{2} \sum_{i=1}^{2}\left((-1)^{i}\left(\alpha_{i j} S_{C_{i}}^{H}+\beta_{i j} I_{C_{i}}^{H}+\gamma_{i j} R_{C_{i}}^{H}\right)\right)}\right) \\
& \frac{d I_{C_{i}}^{V}}{d t}=b_{c_{i}} \beta_{V C_{i}}\left(\frac{\left(I_{C_{i}}^{H}+(-1)^{i}\left(\beta_{12} I_{C_{1}}^{H}-\beta_{21} I_{C_{2}}^{H}\right)\right) S_{C_{i}}^{V}}{N_{C_{i}}^{H}+\sum_{j=1, j \neq i}^{2} \sum_{i=1}^{2}\left((-1)^{i}\left(\alpha_{i j} S_{C_{i}}^{H}+\beta_{i j} I_{C_{i}}^{H}+\gamma_{i j} R_{C_{i}}^{H}\right)\right)}\right)-\lambda_{V C_{i}} I_{C_{i}}^{V}
\end{aligned}
$$

where $i=1,2$.

Table 1 summaries the parameters and their numerical values used in simulation [3, 4].

System (12-16) is solved using MATLAB routine ode 45.

This routine is an implementation of explicit Runge-Kutta $(4,5)$ pair.

Table 1. Parameter definition and values use in numerical simulation

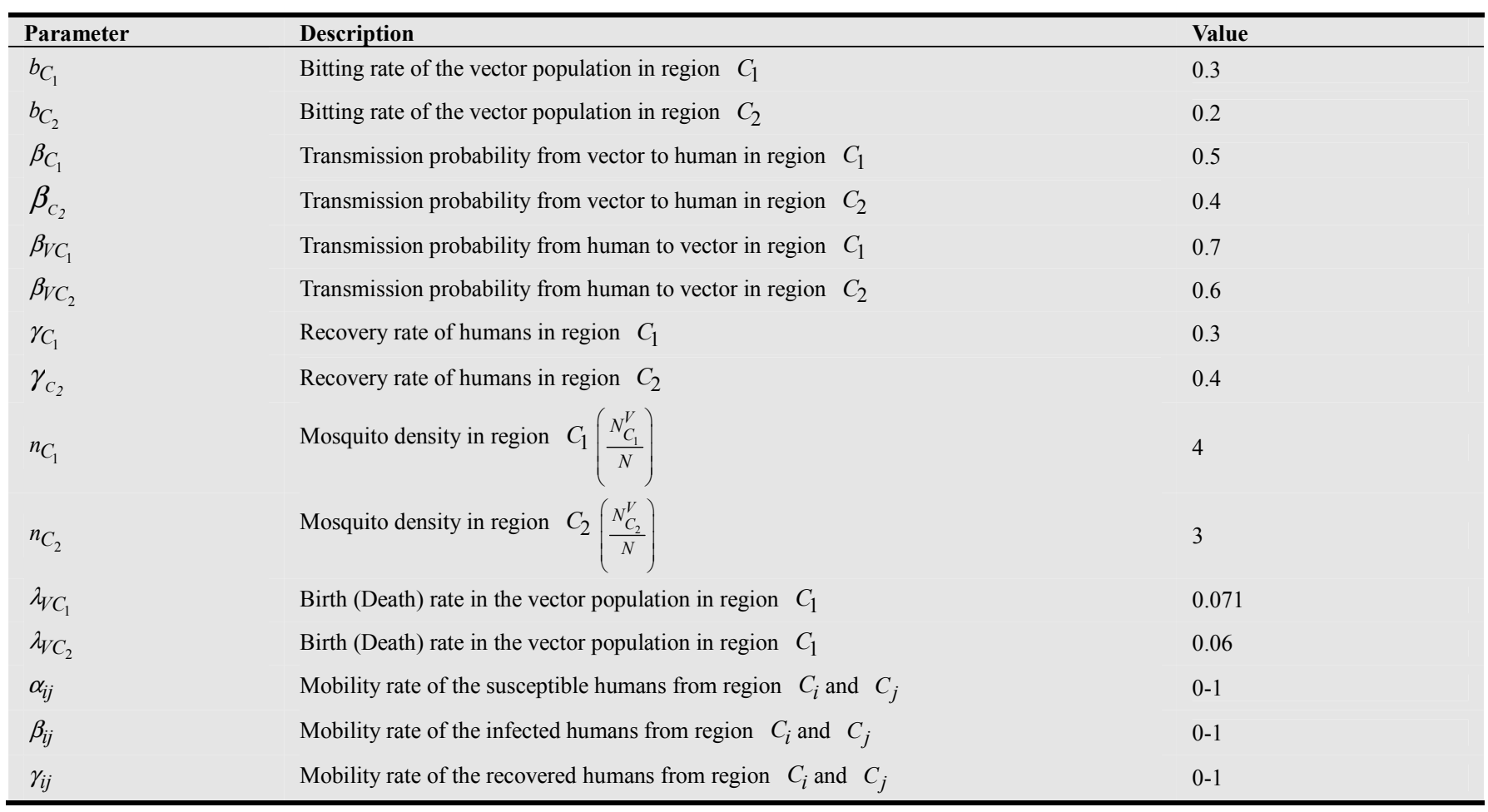

\section{Results and Discussions}

Dengue existing area and human mobility direction are main factors of this mathematical model. We begin by assuming that region $C_{1}$ consists of dengue patients whereas region $C_{2}$ is free of infected human under these conditions, we consider the effect of mobility from region $C_{2}$ to region $C_{1}$. Notice, in Figure 2, that during this time interval, the infected human proportion in region $C_{2}$ (Figure 2f), the infected mosquito proportion in region $C_{2}$ (Figure $2 h$ ) and the susceptible mosquito proportion in region $C_{2}$ (Figure $2 g$ ) remain continuously constant. However the susceptible human proportion in region $C_{2}$ (Figure 2e) decreases because the susceptible humans move to region $C_{1}$.

In Figure 3, again $C_{1}$ initially consists of infected humans while region $C_{2}$ is free of dengue; however in this case humans are only moving from region $C_{1}$ to region $C_{2}$. Notice in Figure $3 f$ that initially the infected human proportion 
increases in region $C_{2}$ followed by an increase in the infected mosquito proportion in region $C_{2}$ (Figure $3 h$ ). In addition, susceptible human proportion in area $C_{1}$ (Figure $3 a$ ), infected human proportion in area $C_{1}$ (Figure $3 b$ ) and

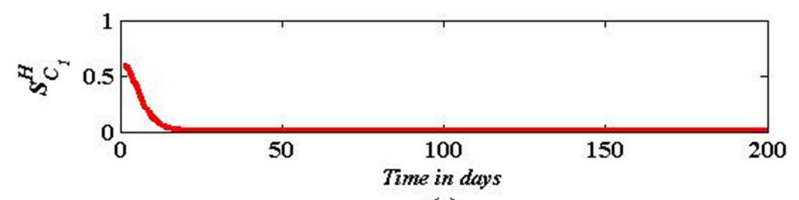

(a)

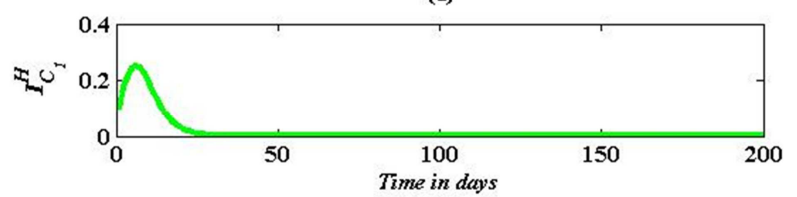

(b)

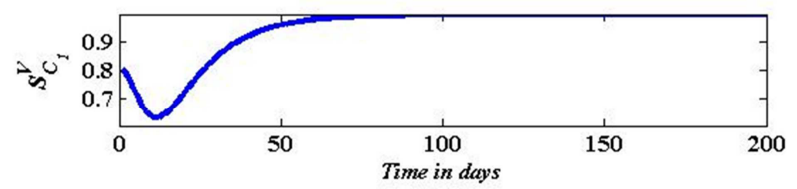

(c)

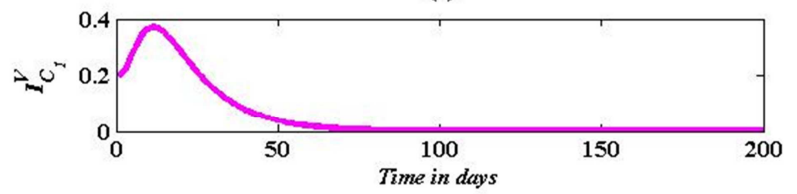

(d) infected mosquito proportion in area $C_{1}$ (Figure $3 d$ ) decrease during this time interval, finally approaching zero. During this same time period, the susceptible mosquito proportion in area $C_{1}$ (Figure 3c) increase to one.

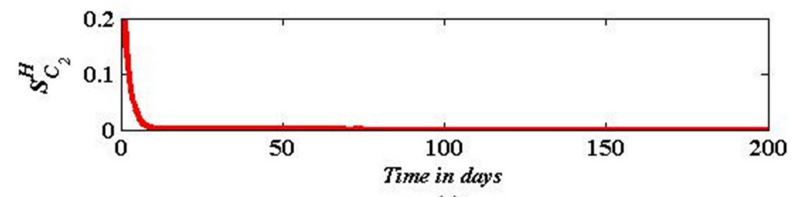

(e)

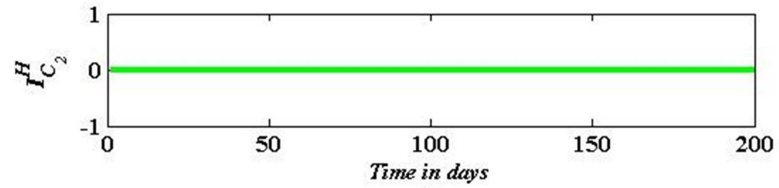

(f)

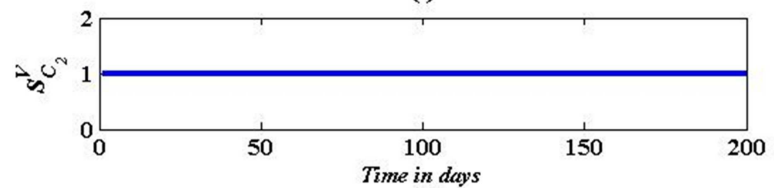

(g)

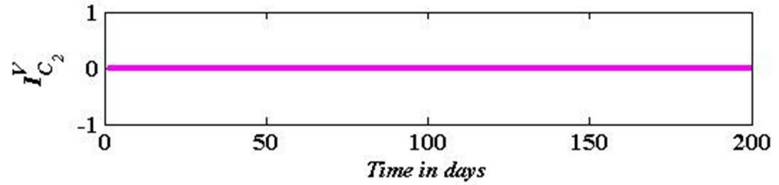

(h)

Figure 2. Dynamics of (a) Susceptible human in region $C_{1}$ (b) Infected human in region $C_{1}$ (c) Susceptible mosquito in region $C_{1}$ (d) Infected mosquito in region (e) Susceptible human in region $C_{2}$ (f) Infected human in region $C_{2}$ (g) Susceptible mosquito in region $C_{2}$ (h) Infected mosquito in region $C_{2}$. Initial condition $S_{C_{1}}^{H}=0.6, I_{C_{1}}^{H}=0.1, R_{C_{1}}^{H}=0.1, S_{C_{1}}^{V}=0.8 I_{C_{1}}^{V}=0.2, S_{C_{2}}^{H}=0.2, I_{C_{2}}^{H}=0, R_{C_{2}}^{H}=0, S_{C_{1}}^{V}=1, I_{C_{2}}^{V}=0$.

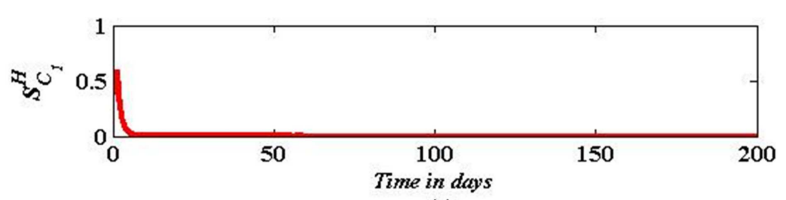

(a)

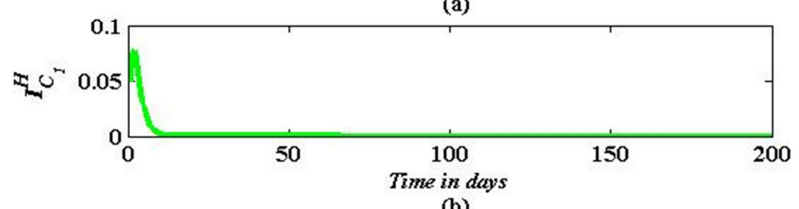

(b)

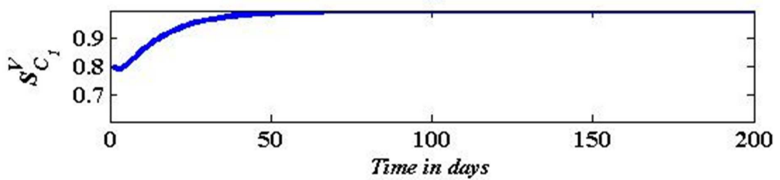

(c)

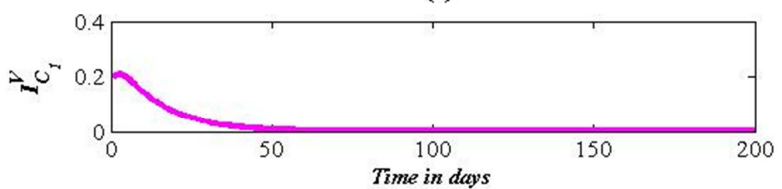

(d)

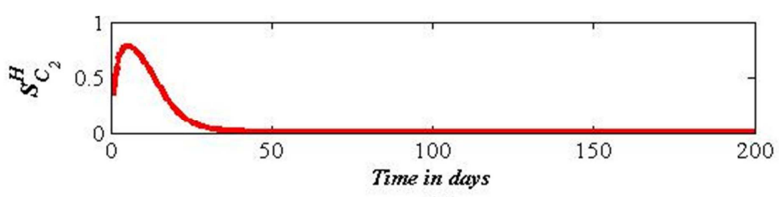

(e)
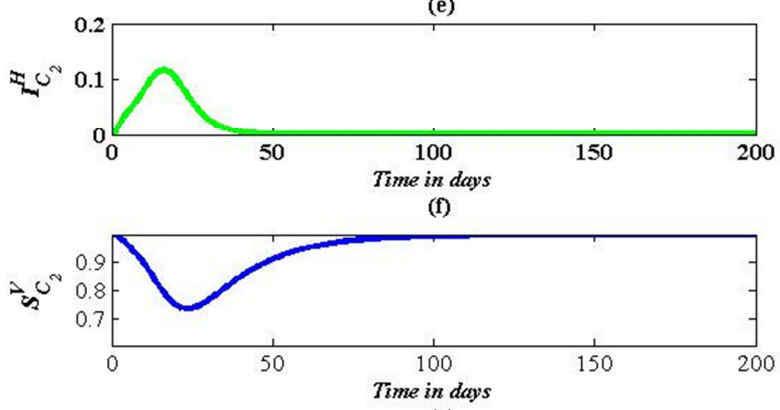

(g)

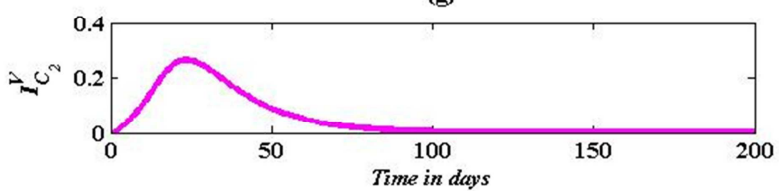

(h)

Figure 3. Dynamics of (a) Susceptible human in region $C_{1}$ (b) Infected human in region $C_{1}$ (c) Susceptible mosquito in region $C_{1}$ (d) Infected mosquito in region (e) Susceptible human in region $C_{2}$ (f) Infected human in region $C_{2}$ (g) Susceptible mosquito in region $C_{2}$ (h) Infected mosquito in region $C_{2}$. Initial condition $S_{C_{1}}^{H}=0.6, I_{C_{1}}^{H}=0.05, R_{C_{1}}^{H}=0, S_{C_{1}}^{V}=0.8 \quad I_{C_{1}}^{V}=0.2, S_{C_{2}}^{H}=0.35, I_{C_{2}}^{H}=0, R_{C_{2}}^{H}=0, S_{C_{1}}^{V}=1, I_{C_{2}}^{V}=0$. 
In the scenario present in Figure 4, initially only humans in region $C_{1}$ may be infected with dengue; however the human population has mobility between regions $C_{1}$ and $C_{2}$. Note that in this case the behavior of both the mosquito and human population in region $C_{1}$ and $C_{2}$ are similar where region $C_{2}$ 's behaviors lag those in region $C_{1}$.

We explore the spread of dengue with both populations in regions $C_{1}$ and $C_{2}$. These results can be seen in Figure 5 .

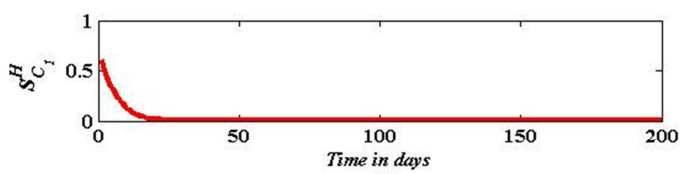

(a)
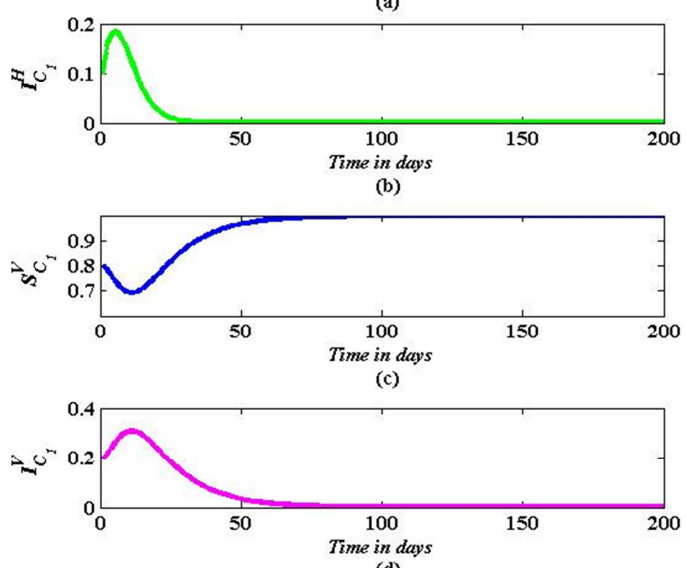

As in Figure 4, behavior of the mosquito and the human populations in both regions are similar with only the parameters affecting what happens initially. In Figure 4, parameter values of $\alpha_{21}, \beta_{21}, \gamma_{21}, \alpha_{12}, \beta_{12}, \gamma_{12}$ are $0.5,0.3,0.6,0.2,0.1,0.2$ respectively. In Figure 5, parameter values of $\alpha_{21}, \beta_{21}, \gamma_{21}, \alpha_{12}, \beta_{12}, \gamma_{12}$ are $0.8,0.4,0.7$, $0.2,0.1,0.2$ respectively. Different between Figure 4 and Figure 5 are initial condition and above parameter values.
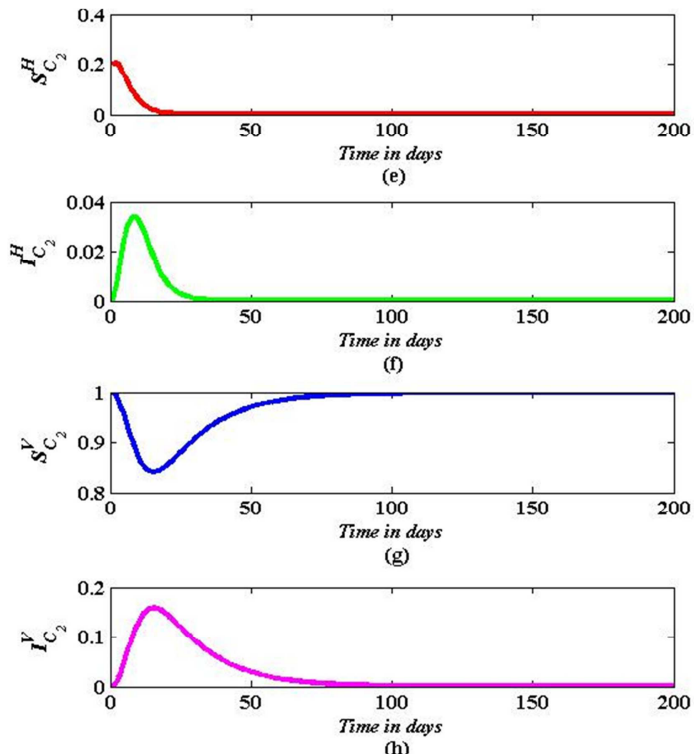

Figure 4. Dynamics of (a) Susceptible human in region $C_{1}$ (b) Infected human in region $C_{1}$ (c) Susceptible mosquito in region $C_{1}$ (d) Infected mosquito in region (e) Susceptible human in region $C_{2}$ (f) Infected human in region $C_{2}$ (g) Susceptible mosquito in region $C_{2}$ (h) Infected mosquito in region $C_{2}$. Initial condition $S_{C_{1}}^{H}=0.6, I_{C_{1}}^{H}=0.1, R_{C_{1}}^{H}=0.1, S_{C_{1}}^{V}=0.8 \quad I_{C_{1}}^{V}=0.2, S_{C_{2}}^{H}=0.2, I_{C_{2}}^{H}=0, R_{C_{2}}^{H}=0, S_{C_{1}}^{V}=1, I_{C_{2}}^{V}=0$.
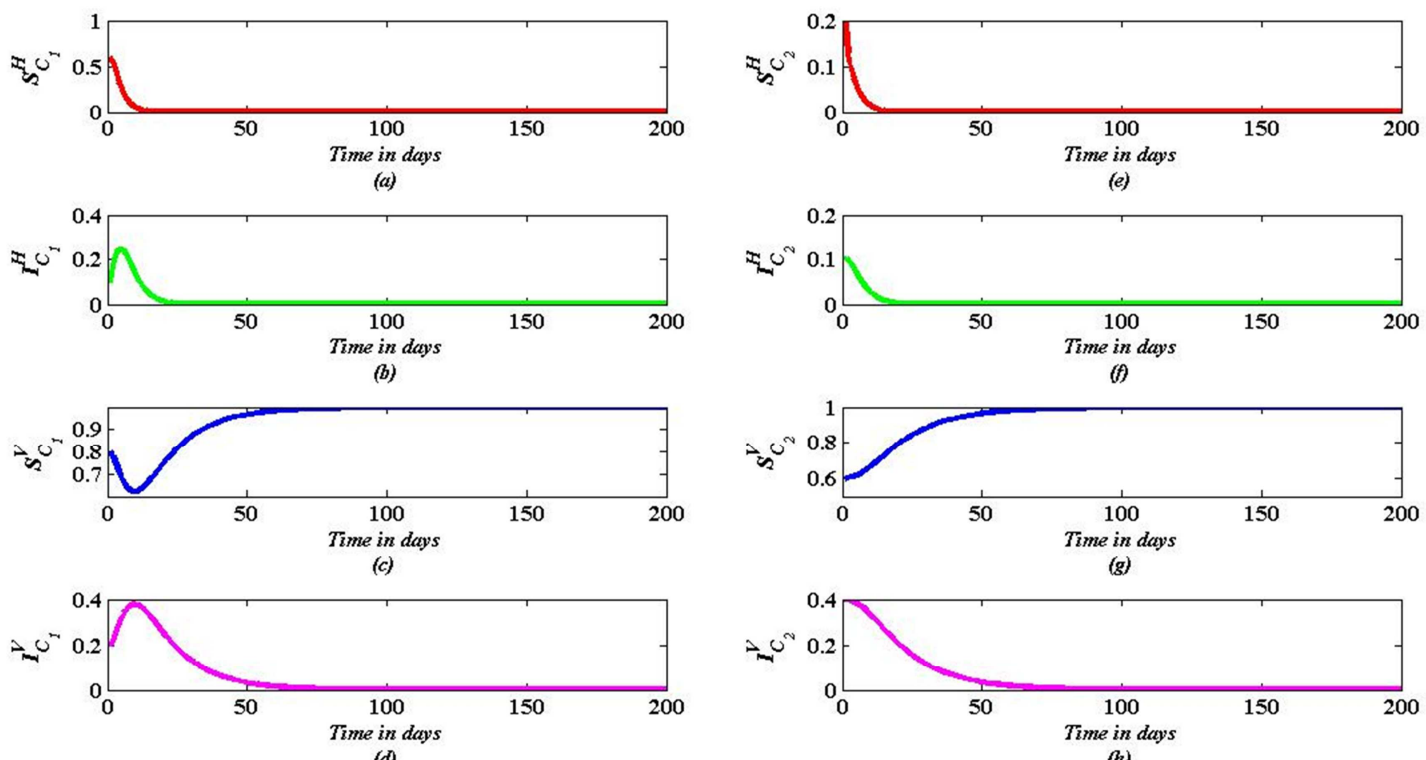

(d)

(h)

Figure 5. Dynamics of (a) Susceptible human in region $C_{1}$ (b) Infected human in region $C_{1}$ (c) Susceptible mosquito in region $C_{1}$ (d) Infected mosquito in region (e) Susceptible human in region $C_{2}$ (f) Infected human in region $C_{2}$ (g) Susceptible mosquito in region $C_{2}$ (h) Infected mosquito in region $C_{2}$. Initial condition $S_{C_{1}}^{H}=0.6, I_{C_{1}}^{H}=0.1, R_{C_{1}}^{H}=0, S_{C_{1}}^{V}=0.8 \quad I_{C_{1}}^{V}=0.2, S_{C_{2}}^{H}=0.2, I_{C_{2}}^{H}=0.1, R_{C_{2}}^{H}=0, S_{C_{1}}^{V}=0.6 I_{C_{2}}^{V}=0.4$. 


\section{Conclusions}

Impact of the human mobility to transmission of dengue disease between two neighboring regions is considered. Here, we consider two regions, namely region $C_{1}$ and region $C_{2}$. We study the impact of human mobility effect to dengue dynamics under four cases. In first three cases, we assume that region $C_{1}$ is dengue existing area where region $C_{2}$ is free from dengue. Initially human mobility exists from region $C_{2}$ to $C_{1}$ followed by the mobility from $C_{1}$ to $C_{2}$ and in the third case mobility exists from region $C_{2}$ to $C_{1}$ and $C_{1}$ to $C_{2}$. In the final case, it is assumed that both the regions $C_{1}$ and $C_{2}$ are affected by the dengue disease and mobility then occurs from the both regions.

It is found that dengue disease can be propagated to disease free area if human mobility exists from dengue existing area to dengue free area. This model can be used to analyse risk of dengue transmission subject to human mobility. For example, according to the simulation we can observe that within 20 days, there is a high risk of dengue and within 50 days dengue disease dies out.

Particularly in large commercial cities, where the visiting population is large, we see that spread of dengue can be affected. Multiple localities and serotypes could complicate the epidemic problem even further. By adding other factors such as climate, socio economic ets can be model further extended.

\section{Acknowledgement}

These studies were made possible by grant from the university of Colombo Research Grant scheme. The work was carried out at Research and Development Center for Mathematical Modeling, Department of Mathematics, University of Colombo, Sri Lanka.

\section{References}

[1] Boston College, Biology Department, Dengue, (2014, July 8), Retrieved from

http://www.bc.edu/schools/cas/biology/research/infect/dengue. html.

[2] M. Derouich, and A. Boutayeb, "Dengue fever: Mathematical Modeling and Computer Simulation," Applied Mathematics and Computation, vol. 177, No 2, 2006, pp 528-544.

[3] P. Puntani, "Transmission Model for Dengue Disease with and without the Effect of Extrinsic Incubation Period," KMITL Sci. Tech. J., vol. 6, No 2, 2006, pp 74-82.

[4] M. Derouich, A. Boutayeb, and E. H. Twizell, "A model of dengue fever," BioMedical Engineering OnLine, vol. 2, No 4, 2003, pp 1-10.

[5] National Institute for Allergy and Infectious Disease, Dengue
Fever, (2014, July 8), Retrieved from http://www.niaid.nih.gov/topics/DengueFever/Research/Basic Research/pages/howdenguematures.aspx

[6] M. Choisy, J. F. Guegan and P. Rohani, "Mathematical Modeling of infectious Diseases Dynamics," Encyclopedia of infectious Diseases: Modern Methodologies.

[7] R. Sinden, "Malaria, Mosquitoes and the Legacy of Ronald Ross," Bull World Health Organ, vol. 85, No. 11, 2007, pp 894-896.

[8] S. T. R. Pinho, C. P. Ferreira, L. Esteva, F. R. Barreto, V. C. Morato e Silva and M. G. L. Teixeria, "Modelling the Dynamics of Dengue Real Epidemics," Philosophical Transactions of the Royal Society A, vol. 368, 2010, pp 5679-5693.

[9] L. B. L. Santos, M. C. Costa, S. T. R. Pinho, R. F. S. Andrade, F R. Barreto, M. G. Teixeira and M. L. Barreto, "Periodic Forcing in a Three-Level Cellular Automata Model for a Vector-Transmitted Disease," Physical. Review. E, vol. 80, 2009, pp 016102-016109.

[10] H. M. Yang, and C. P. Ferrerira, "Assessing the Effects Vector Control on Dengue Transmission," Applied Mathematics and Computation, vol. 198, 2008, pp 401-413.

[11] N. A. Maidana, and H. M. Yang, "Describing the Geographic Spread of Dengue Disease by Traveling Waves," Mathematical Biosciences, vol. 215, 2008, pp 64-77.

[12] History of Dengue, (2016), Dengue Virus Net, http://www.Denguevirusnet.com/history-of-Dengue.html.

[13] Dengue and Severe dengue, (2016, April), WHO, http://www.who.int/mediacentre/factsheets/fs117/en/.

[14] W. P. T. M. Wickrmaarachchi, S. S. N. Perera, S. Jayasinghe, and P. Kariyawasam, "The influence of the human mobility for dengue disease transmission in Urban Colombo and bordering areas of Colombo: A cross wavelet approach," International Conference of Eastern University Sri Lanka, 2013, pp 24.

[15] W. P. T. M. Wickrmaarachchi, S. S. N. Perera, S. Jayasinghe, P. Kariyawasam, and P. Kariyawasam "Mathematical Modeling and Dengue: An analysis of Incidence of Dengue in Urban Colombo using Wavelet Approach," International Conference on Public Health Innovations, National Institute of Health Sciences, Sri Lanka, 2013, pp 49.

[16] Sunmi Lee, and Carlos Castillo-Chavez "The role of residence time in two-patch dengue transmission dynamics and optimal strategies," Journal of Theoretical Biology, vol. 374, 2015, pp 152-164.

[17] Steven T. Stoddard, Amy C. Morrison, Gonzalo M. Vazquez-Prokopec, Valerie Paz Soldan, Tadeusz J. Kochel, Uriel Kitron, John P. Elder, and Thomas W. Scott, "The role of Human Movement in the transmission of Vector-Borne Pathogens," PLOS Neglected Tropical Disease, vol. 3, No 7, 2009, pp e481

[18] Sumith Pathirana, Mosato Kawabata, "Study of potential risk of dengue disease outbreak in Sri Lanka using GIS and statistical modeling," Southern Cross University, vol 8, 2009, pp 8-17. 